\title{
Long-term follow-up after bypass surgery or coronary stenting in elderly with multivessel disease
}

\author{
M. E. Gimbel · L. M. Willemsen · M. C. Daggelders · J. C. Kelder · T. Oirbans · K. F. Beukema · E. J. Daeter · \\ J. M. ten Berg
}

Published online: 24 April 2020

(C) The Author(s) 2020

\begin{abstract}
Background We sought to compare long-term followup of coronary artery bypass grafting (CABG) with percutaneous coronary intervention (PCI) in elderly patients with left main or multivessel disease, hypothesising that completeness of revascularisation and severity of coronary artery disease are predictors of adverse outcomes.

Methods Patients aged $\geq 75$ years with multivessel disease or left main disease who underwent PCI or CABG between 2012-2016 were included in this retrospective cohort study. Baseline characteristics from the index procedure were collected. Severity of coronary artery disease and completeness of revascularisation were assessed. Primary outcome was all-cause mortality, in addition we captured major adverse cardiac and cerebral events, bleedings, recurrent angina and new onset atrial fibrillation.

Results A total of 597 patients were included. Median follow-up was 4 years (interquartile range 2.8-5.3 years). At baseline, patients in the PCI group more often had a previous medical history of CABG and more frequently underwent an urgent procedure compared with patients in the CABG group. Mortality at 5-year follow-up was significantly higher in patients who underwent PCI compared with CABG (39.9\% vs $25.4 \%$, $p<0.001)$. Furthermore, acute coronary syndrome (ACS), repeat revascularisation and recurrent angina occurred more frequently after PCI, while occurrence of bleedings and new onset atrial fibrillation were
\end{abstract}

\footnotetext{
M.E. Gimbel and L.M. Willemsen shared first authors.

M. E. Gimbel $(\bowtie) \cdot$ L. M. Willemsen · M. C. Daggelders ·

J. C. Kelder · T. Oirbans · K. F. Beukema · E. J. Daeter ·

J. M. ten Berg

Department of Cardiology and Cardiothoracic Surgery, St.

Antonius Hospital, Nieuwegein, The Netherlands

m.gimbel@antoniusziekenhuis.nl
}

more frequent after CABG. Neither completeness of revascularisation nor severity of coronary artery disease was a predictor for any of the outcomes.

Conclusion Long-term mortality was higher in elderly patients with multivessel disease undergoing PCI compared with CABG. In addition, patients undergoing PCI had a higher risk of ACS, repeat revascularisation and recurrent angina.

Keywords Revascularisation - Coronary artery disease $\cdot \mathrm{CABG} \cdot \mathrm{PCI}$

\section{Background}

Coronary artery bypass grafting (CABG) has long been the standard of care for patients with left main or multivessel disease. However, results of percutaneous coronary intervention (PCI) have been improved by

\section{What's new}

- Patients of 75 years or older with multivessel or left main disease have a significantly higher 5-year mortality when treated by percutaneous coronary intervention (PCI) compared with coronary artery bypass grafting (CABG) with an adjusted hazard ratio of 1.59.

- Recurrent acute coronary syndrome, repeat revascularisation and recurrent angina all occurred significantly more frequently in these elderly patients who underwent PCI compared with CABG, while new onset atrial fibrillation and bleeding occurred significantly more often after CABG than after PCI.

- Completeness of revascularisation appeared not to be an independent predictor of adverse outcomes in this patient population. 
better stents and more potent $\mathrm{P}_{2} \mathrm{Y}_{12}$-inhibitors. Therefore, the European Society of Cardiology (ESC) guideline for management of myocardial revascularisation now recommends either CABG or PCI based on individual decision making by the local heart team, taking into consideration operation risk, complexity of underlying coronary artery disease, intracardiac and extracardiac factors and local expertise. Furthermore, it is emphasised that achieving complete revascularisation is pivotal [1]. The ESC guideline does not advise on which revascularisation strategy is preferred in elderly patients in comparison with younger patients, probably because the optimal revascularisation treatment in elderly is unknown. PCI is less invasive with shorter hospital stay and earlier return to daily activities compared with CABG. This is particularly relevant for the elderly, in whom physical recovery after CABG procedures is substantially prolonged compared with younger patients [2]. Several observational studies have been conducted comparing PCI and CABG in the elderly ( $\geq 75$ years) with multivessel disease and/or left main disease [3-6]. These studies found CABG to be associated with a significantly lower risk for target vessel revascularisation but found no significant difference in all-cause death. These studies were performed in patients treated with first generation drugeluting stents and dual antiplatelet therapy consisting of aspirin with clopidogrel. Also, patients treated with PCI or CABG were not similar with respect to completeness of revascularisation and complexity of coronary artery disease. Therefore, aim of this study is to compare CABG with PCI in elderly ( $\geq 75$ years) patients with multivessel or left main disease, considering completeness of revascularisation and severity of coronary artery disease.

\section{Methods}

\section{Study design}

We conducted a retrospective, single-centre cohort study in the St. Antonius hospital, the Netherlands. All patients aged $\geq 75$ years with multivessel disease or left main disease who underwent revascularisation between January 1st, 2012 and December 31st, 2016 were included. Patients underwent revascularisation either by PCI or CABG, which was decided by a multidisciplinary heart team consisting of an interventional cardiologist and a cardiac surgeon. Patients who presented with ST-segment elevation myocardial infarction or who underwent emergency revascularisation were excluded. All patients were treated according to the applicable guidelines at that moment. The surgical technique for CABG, the approaches used for stent implantation, and medication regimen post revascularisation were left to the discretion of the treating physician. Patients were included if they had at least one year follow-up after the index procedure. Patients with follow-up in other hospitals were sent a question- naire inquiring about recurrent revascularisation, myocardial infarction, cerebral vascular accident (CVA), bleeding, angina or cardiac hospitalisation. Indicated events were verified by assessing patients' medical records. The study was conducted according to the principles of the Declaration of Helsinki and in accordance with the Medical Research Involving Human Subjects Act. A waiver for written informed consent was provided by the local ethics committee.

\section{Data collection}

Information was obtained from patients' medical records or retrieved from patients' general practitioner. Collected patients' characteristics included sex, age, diabetes mellitus, creatinine ( $\mathrm{mmol} / \mathrm{l})$, history of CABG, history of atrial fibrillation, location of lesions, completeness of revascularisation (determined by location of lesions and revascularised vessels through reviewing the pre-procedural angiogram, procedural angiogram (PCI) and revascularisation reports (PCI and CABG) by at least two qualified researchers), type of stent implanted (bare-metal stent, second generation drug-eluting stent, bioresorbable vascular scaffold (BVS)), urgency of procedure and Euroscore I. Also, the national mortality register was consulted.

\section{Definitions}

A stenosis of $\geq 70 \%$ or fractional flow reserve measurement $<0.80$ was considered significant in a coronary vessel of $\geq 2.0 \mathrm{~mm}$ in diameter. A left main stenosis was considered significant when $\geq 50 \%$. Multivessel disease was presence of a significant stenosis in the left main or at least two major coronary arteries. A procedure was considered elective when it was scheduled and performed on patients with stable coronary artery disease, urgent when it was performed in context of an acute coronary syndrome (ACS) and emergency when it was performed immediately because of the acute nature of the medical condition and increased morbidity or mortality associated with temporary delay in treatment [7]. Completeness of revascularisation was determined as treatment of all significant lesions. ACS was defined according to the Fourth Universal Definition of myocardial infarction or unstable angina [8]. CVA was described as acute new neurological deficit by ischaemic stroke which lasted $>24 \mathrm{~h}$ or ended in death within $24 \mathrm{~h}$, excluding haemorrhagic CVAs. Repeat revascularisation was defined as revascularisation with either PCI or CABG unless index treatment was scheduled as a staged procedure. In the absence of questionnaires, the following outcome measures were chosen to provide an indication of quality of life: recurrent angina, cardiac rehospitalisation and new onset atrial fibrillation. Recurrent angina was classified according to the Canadian Cardiovascular Society of Angina Grading scale. Angina 


\title{
Advertisement placed here.
}

\author{
ces bohn \\ CL van loghum
}

Houten 2020 


\title{
Advertisement placed here.
}

\author{
ces bohn \\ CL van loghum
}

Houten 2020 


\title{
Advertisement placed here.
}

\author{
ces bohn \\ CL van loghum
}

Houten 2020 


\title{
Advertisement placed here.
}

\author{
ces bohn \\ CL van loghum
}

Houten 2020 
definitely provoked by other causes e.g. anaemia or tachycardia was excluded. Angina was further subdivided into documented ischaemia, which included either positive electrocardiogram exercise testing, stress imaging or when adjustment of pharmaceutical therapy for angina relieved the symptoms. Cardiac rehospitalisation was specified as readmission after the procedure for any cardiac cause, e.g. heart failure or atrial fibrillation. New onset atrial fibrillation was captured when it occurred after the procedure and remained after discharge or presented post-discharge. Bleeding was classified according to Bleeding Academic Research Consortium (BARC) criteria, we captured BARC bleeding type 3 and 5 [9].

\section{Outcome}

Primary outcome was all-cause mortality. We also captured ACS, CVA, recurrent angina, repeat revascu- larisation, cardiac rehospitalisation, new onset atrial fibrillation and bleeding events.

\section{Statistical analysis}

Baseline characteristics were compared using Student's t-test or Mann-Whitney U test for continuous variables and chi-squared test for binary variables. Continuous data were expressed as mean \pm standard deviation (SD). Categorical variables were described as frequencies and percentages. Unadjusted primary and secondary outcomes were presented as KaplanMeier curves, differences were assessed by using the log-rank test. Risk-adjusted hazard ratios (aHR) with 95\% confidence intervals (CI) were estimated by Cox proportional hazard regression. Baseline variables with a $p$-value $<0.100$ in the univariate analysis were included in the multivariate analysis. $P<0.05$ was considered statistically significant.

\begin{tabular}{|c|c|c|c|c|c|}
\hline \multirow{33}{*}{$\begin{array}{l}\text { Table } 1 \\
\text { teristics }\end{array}$} & \multirow[t]{33}{*}{ Baseline charac- } & Characteristics & $\mathrm{PCl}(N=346)$ & $\mathrm{CABG}(N=251)$ & $P$-value \\
\hline & & Male gender- $N(\%)$ & $225(65)$ & $183(73)$ & 0.041 \\
\hline & & Age-year mean $\pm S D$ & $80 \pm 3.9$ & $79 \pm 3.4$ & $<0.001$ \\
\hline & & Age $\geq 80-N(\%)$ & $186(54)$ & $98(39)$ & $<0.001$ \\
\hline & & Diabetes-N(\%) & $93(27)$ & $75(30)$ & 0.433 \\
\hline & & Creatinine $\mu \mathrm{mol} / /$ mean $\pm S D$ & $109 \pm 85$ & $102 \pm 42$ & 0.246 \\
\hline & & Creatinine $\geq 200 \mu \mathrm{mol} / \mathrm{l}-N(\%)$ & $10(3.2)$ & $6(2.4)$ & 0.573 \\
\hline & & History of $\mathrm{CABG}-N(\%)$ & $71(21)$ & $14(5.6)$ & $<0.001$ \\
\hline & & History of $\mathrm{AF}-\mathrm{N}(\%)$ & $43(12)$ & $23(9)$ & 0.125 \\
\hline & & Status elective $-N(\%)$ & $260(75)$ & $208(83)$ & 0.024 \\
\hline & & Status urgent- $N(\%)$ & $86(25)$ & $43(17)$ & \\
\hline & & Complete revascularisation $-N(\%)$ & $102(30)$ & $179(71)$ & $<0.001$ \\
\hline & & Coronary artery disease & & & \\
\hline & & $\mathrm{LAD}>70 \%-N(\%)$ & $263(76)$ & $233(93)$ & $<0.001$ \\
\hline & & $\mathrm{RCx}>70 \%-N(\%)$ & $231(67)$ & $192(77)$ & 0.010 \\
\hline & & $\mathrm{RCA}>70 \%-N(\%)$ & $236(68)$ & $192(77)$ & 0.027 \\
\hline & & $\mathrm{LM}>50 \%-N(\%)$ & $48(14)$ & $70(28)$ & $<0.001$ \\
\hline & & Single LM disease $-N(\%)$ & $12(3.5)$ & $3(1.2)$ & 0.080 \\
\hline & & $\mathrm{LM}+1 \mathrm{VD}-N(\%)$ & $20(5.8)$ & $14(5.6)$ & 0.916 \\
\hline & & $\mathrm{LM}+2 \mathrm{VD}-\mathrm{N}(\%)$ & $4(1.2)$ & $30(12)$ & $<0.001$ \\
\hline & & $\mathrm{LM}+3 \mathrm{VD}-N(\%)$ & $12(3.5)$ & $23(9.2)$ & 0.003 \\
\hline & & $2 \mathrm{VD}-N(\%)$ & $228(66)$ & $69(28)$ & $<0.001$ \\
\hline & & $3 \mathrm{VD}-N(\%)$ & $70(20)$ & $112(45)$ & $<0.001$ \\
\hline & & $\mathrm{PCl}$ characteristics & & & \\
\hline & & $\mathrm{DES}-N(\%)$ & $309(89)$ & & \\
\hline & & $\mathrm{BMS}-N(\%)$ & $27(7.8)$ & & \\
\hline & & BVS-N $(\%)$ & $2(0.6)$ & & \\
\hline & & Balloon-N(\%) & $81(23)$ & & \\
\hline & & Number of stents mean $\pm \mathrm{SD}$ & $1.71 \pm 1.0$ & & \\
\hline & & CABG characteristics & & & \\
\hline & & Euroscore I mean $\pm \mathrm{SD}$ & & $8.6(7.7)$ & \\
\hline & & LIMA-N $(\%)$ & & $235(94)$ & \\
\hline & & $\begin{array}{l}A F \text { atrial fibrillation, } B M S \text { bare-meta } \\
D E S \text { drug-eluting stent, } L A D \text { left an } \\
R C A \text { right coronary artery, } R C \times \text { ramu }\end{array}$ & $\begin{array}{l}\text { esorbable vasc } \\
\text { ig artery, } L M \text { le } \\
\text { ery, } S D \text { standar }\end{array}$ & $\begin{array}{l}\text { Id, } C A B G \text { coronar } \\
\text { PI percutaneous } \\
\text {, VD vessel disea }\end{array}$ & $\begin{array}{l}\text { ypass grafting; } \\
\text { intervention, }\end{array}$ \\
\hline
\end{tabular}




\section{Results}

We included a total of 597 patients; 346 in the PCI group and 251 in the CABG group. Median follow-up period was 4 years (interquartile range [IQR] 2.8-5.3 years). Baseline characteristics are presented in Tab. 1. Patients who underwent PCI were older; $54 \%$ of patients in the PCI group were aged $\geq 80$ vs $39 \%$ in the CABG group $(p<0.001)$. Patients in the PCI group more frequently had a previous medical history of CABG (21 vs $5.6 \%, p<0.01)$ and more often needed urgent revascularisation (25 vs $17 \%, p=0.024$ ) compared with patients in the CABG group. Patients who underwent CABG were more often male (73 vs $65 \%$, $p=0.041$ ), had more coronary segments involved, and had left main disease more frequently (28 vs $14 \%$, $p<0.01)$ compared with patients who underwent PCI. Complete revascularisation was more frequently achieved in patients undergoing CABG than in patients undergoing PCI (71 vs $30 \%, p<0.01$ ). Incidence of diabetes mellitus and serum creatinine levels were similar in both groups. Among PCI patients, the majority received a drug-eluting stent (89\%). Most CABG patients received a left internal mammary artery graft (94\%). Mean Euroscore I in the CABG group was 8.6. Loss to follow-up of the study population is presented in Fig. 1. For the primary outcome we checked the national mortality register. Therefore, only 19 patients were lost to follow-up.

\section{Mortality}

The unadjusted analyses showed a significantly higher long-term mortality rate after PCI than after CABG (39.9\% vs $25.4 \%, p=0.001$; Fig. 2). Cox-regression analysis revealed older age, higher creatinine and left main disease to be independent predictors of longterm mortality. After adjustment for these predictors, 5-year mortality remained significantly higher after PCI (aHR 1.59 [95\% CI 1.10-2.28], $p=0.013$ ).

\section{Individual outcomes}

After adjustment for the concerning independent predictors, recurrent ACS, consisting of myocardial infarction in $73 \%$ of cases (aHR 2.20 [95\% CI 1.23-3.96], $p=0.008$ ), repeat revascularisation (aHR $2.54[95 \%$ CI 1.36-4.73], $p=0.003$ ) and recurrent angina (aHR 1.63 [95\% CI 1.15-2.33], $p=0.007$ ), all occurred more frequently in patients who underwent PCI compared with CABG. On the other hand, new onset atrial fibrillation (aHR 0.40 [95\% CI $0.20-0.79], p=0.008$ ) and bleeding (aHR 0.10 [95\% CI 0.02-0.53], $p=0.007$ ) occurred significantly more often in patients who underwent CABG. The incidence of CVA and cardiac rehospitalisation was comparable between both groups (Fig. 3).

\section{Recurrent angina}

Recurrent angina during first year after index procedure developed more often in PCI patients compared with CABG patients $(24.4$ vs $9.2 \%, p<0.001)$. The difference between the two groups, however, decreased during follow-up (Fig. 4). Recurrent angina was caused by documented ischaemia in $72 \%$ of cases, and differed significantly in favour of CABG (37.9 vs $20.2 \%, p<0.012)$.

\section{Discussion}

In this large and unselected registry of patients aged 75 years or older with multivessel disease or left main disease treated with PCI or CABG, we identified higher mortality after PCI than after CABG. In addition, we found patients undergoing PCI to have higher risk of

Fig. 1 Flowchart followup. $C A B G$ coronary artery bypass grafting, FU followup, $\mathrm{PCl}$ percutaneous coronary intervention

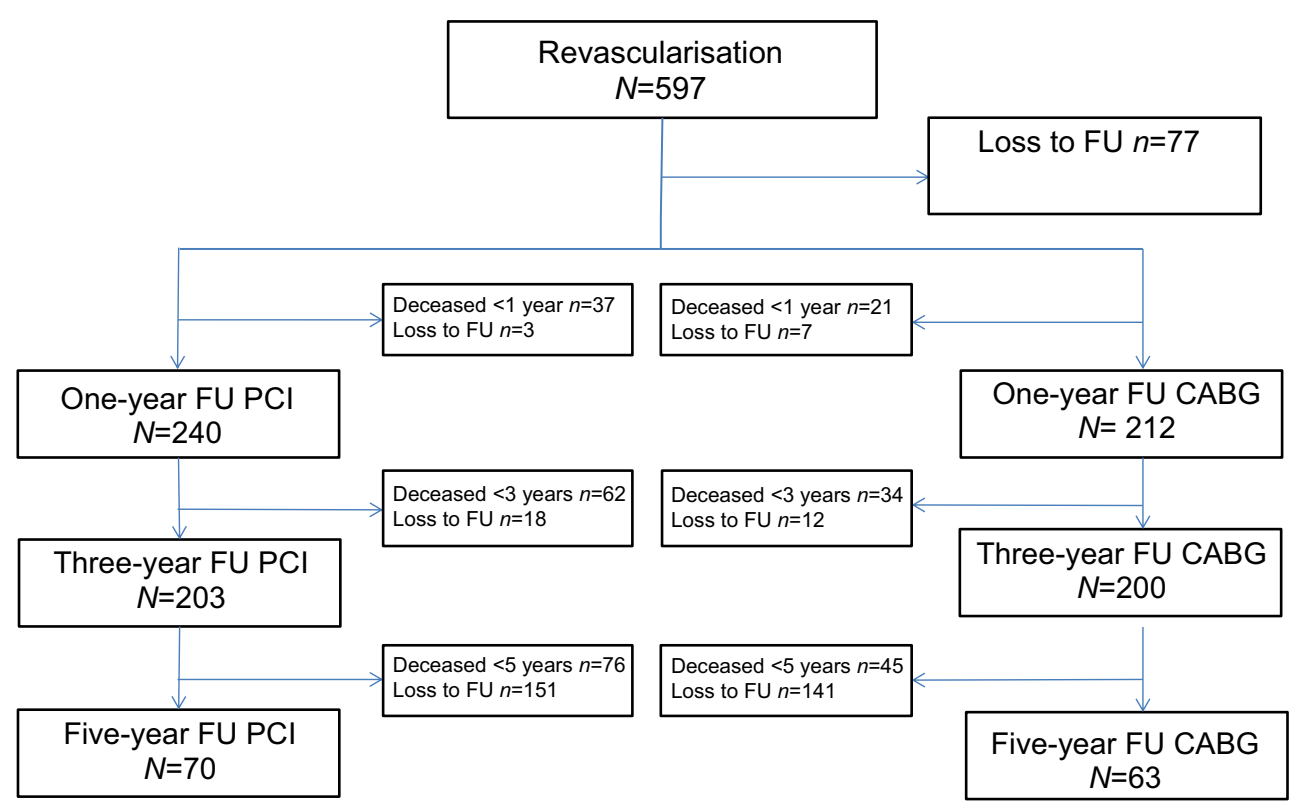


Fig. 2 Kaplan-Meier survival curve for primary outcome. CABG coronary artery bypass grafting, $\mathrm{PCl}$ percutaneous coronary intervention

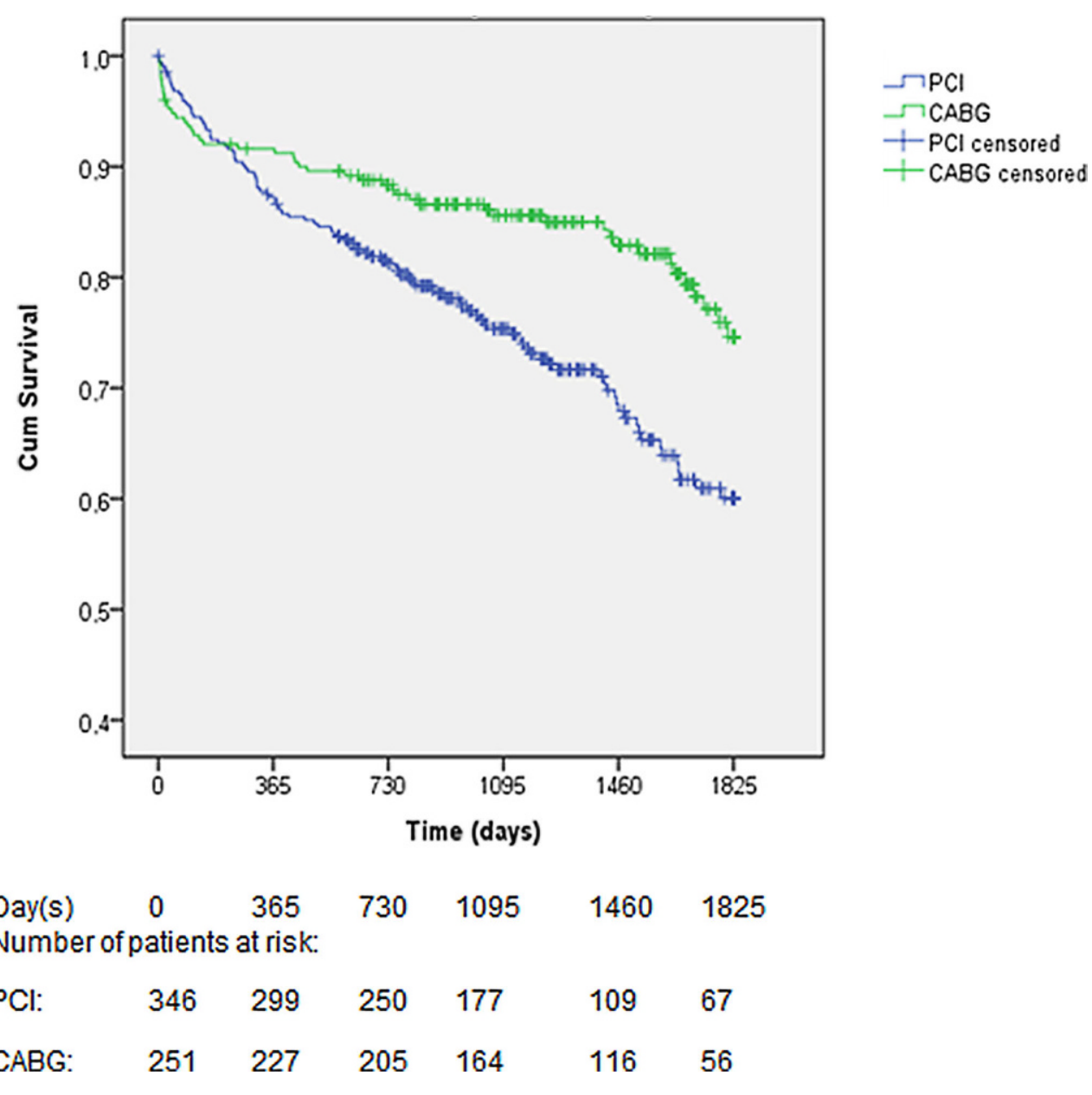

\begin{tabular}{|c|c|c|c|c|c|c|c|c|}
\hline & PCl Event & $\%$ & CABG Event & $\%$ & $\mathrm{HR}[95 \% \mathrm{Cl}]$ & $p$-value & & \\
\hline Mortality & 105 & 30.3 & 47 & 18.7 & $1.59[1.10-2.28]$ & 0.013 & & $1-1$ \\
\hline Acute coronary syndrome & 45 & 16.1 & 16 & 6.7 & $2.20[1.23-3.96]$ & 0.008 & & $\longmapsto$ \\
\hline Myocardial infarction & 33 & 11.8 & 12 & 5.0 & $2.15[1.09-4.25]$ & 0.028 & & $\longmapsto$ \\
\hline Cerebrovascular accident & 8 & 2.9 & 8 & 3.3 & $0.96[0.36-2.55]$ & 0.928 & & $=$ \\
\hline Repeat revascularisation & 35 & 12.5 & 14 & 5.8 & $2.54[1.36-4.73]$ & 0.003 & & $\longmapsto$ \\
\hline Recurrent angina & 102 & 36.4 & 53 & 22.1 & $1.63[1.15-2.33]$ & 0.007 & & $\mapsto-1$ \\
\hline Documented ischaemia & 76 & 27.1 & 35 & 4.6 & $1.76[1.13-2.74]$ & 0.012 & & $\mapsto$ \\
\hline Cardiac rehospitalisation & 107 & 38.2 & 64 & 26.7 & $1.09[0.80-1.49]$ & 0.600 & & $1=-1$ \\
\hline Atrial fibrillation de novo & 12 & 4.3 & 27 & 11.3 & $0.40[0.20-0.79]$ & 0.008 & $\longmapsto$ & \\
\hline \multirow[t]{2}{*}{ Bleeding BARC 3 and 5} & 2 & 0.7 & 14 & 3.1 & $0.10[0.02-0.53]$ & 0.007 & $\longmapsto$ & \\
\hline & & & & & & & $\begin{array}{llllll}0.03 & 0.06 & 0.12 & 0.25 & 0.50 & 0 .\end{array}$ & $0.03 \quad 0.004 .00$ \\
\hline $\mathbf{a}$ & & & & & & & b & \\
\hline
\end{tabular}

Fig. 3 Five-year outcomes after PCI and CABG. BARC Bleeding Academic Research Consortium, CABG coronary artery bypass graft, $\mathrm{Cl}$ confidence interval, $\mathrm{HR}$ hazard ratio, $\mathrm{PCl}$ percutaneous coronary intervention 
Fig. 4 Recurrent angina classified according to the Canadian Cardiovascular Society of Angina grading scale: Grade 0 asymptomatic/absent angina; Grade I angina only with strenuous exertion; Grade II angina with moderate exertion; Grade III angina with mild exertion; Grade IV angina at rest. CCN Cardiac Care Network of Ontario

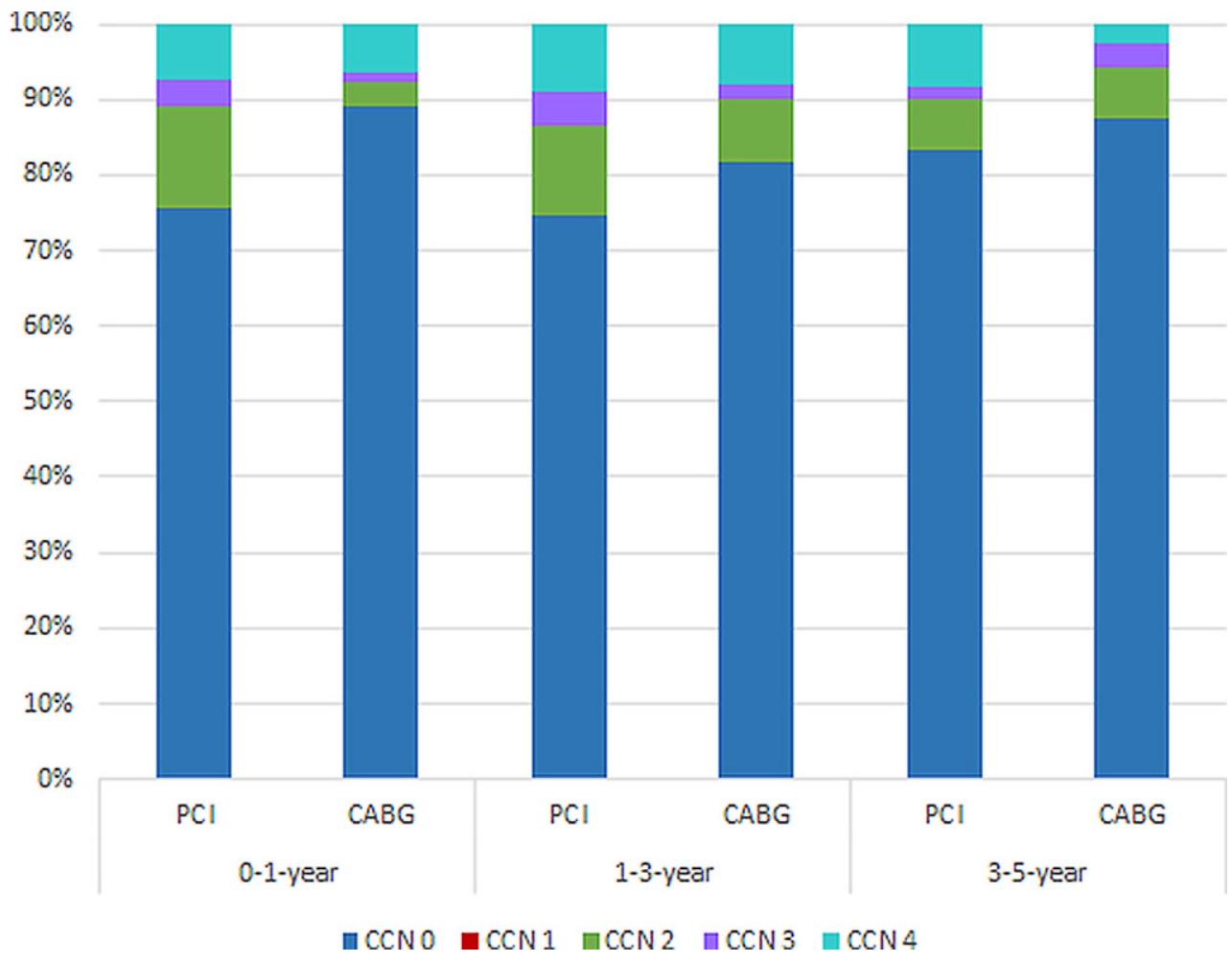

with the literature where target vessel revascularisation and heart failure hospitalisations occurred significantly less frequently in the CABG group compared with the PCI group [6, 11]. This difference in repeat revascularisation and hospitalisation could be explained by more frequently occurring failure of revascularisation (restenosis) after PCI than after CABG (graft failure) or by more complete revascularisation after CABG than after PCI. However, the latter is not corroborated by our study, where incomplete revascularisation was not an independent predictor of death or major adverse cardiac and cerebral events.

The 2018 ESC guideline on myocardial revascularisation recommends prioritising completeness of revascularisation when deciding between CABG and PCI, based on a meta-analysis of 35 randomised controlled trials and observational studies [12]. Complete revascularisation was associated with reduced longterm mortality compared with incomplete revascularisation which was observed both after CABG and after PCI. However, evidence concerning revascularisation in octogenarians showed conflicting results [13, 14]. In these elderly patients, it is suggested that complete revascularisation is not necessary to provide good long-term prognosis. This is supported by Généreux et al. who, based on SYNTAX score, identified $70 \%$ completeness of revascularisation to be sufficient to provide comparable long-term prognosis to $100 \%$ completeness of revascularisation [15].

A strength of this study is the consistency and uniformity of both procedures during the study period in our centre, e.g. same decision making process in the 
heart team, similar and contemporary revascularisation methods (performed by the same surgeons and cardiologists), and the medical treatment after both revascularisation methods was according to the same hospital protocols. This suggests that effects found in this study, are truly attributable to the revascularisation method, while this may be different in multicentre studies. In addition, all coronary angiograms were reviewed and compared with revascularisation reports to ascertain completeness of revascularisation.

Some important limitations of this study should also be discussed. First, the retrospective design of the study may have resulted in selection bias allocating patients to one of the two revascularisation strategies. By using adjustment through Cox-regression we tried to correct for the differences in baseline variables. However, we should take into account that this still could have had an influence on the results. In addition, in both groups we may have included patients who had an absolute contraindication for the other revascularisation strategy. Second, we were unable to measure quality of life. Quality of life is an important measure, especially at advanced age, and could differ between PCI and CABG patients, as the recovery and rehabilitation period after CABG is longer and more intense than after PCI. However, we evaluated recurrent angina and rehospitalisation as substitute outcomes, capturing, in our view, important aspects of quality of life.

To conclude, in this observational study, long-term mortality was higher in elderly patients of 75 years or older with multivessel disease undergoing PCI as compared with CABG. In addition, patients undergoing PCI had a higher risk of ACS, repeat revascularisation and recurrent angina.

Conflict of interest M.E. Gimbel, L.M. Willemsen, M.C. Daggelders, J.C. Kelder, T. Oirbans, K.F. Beukema, E.J. Daeter and J.M. ten Berg declare that they have no competing interests.

Open Access This article is licensed under a Creative Commons Attribution 4.0 International License, which permits use, sharing, adaptation, distribution and reproduction in any medium or format, as long as you give appropriate credit to the original author(s) and the source, provide a link to the Creative Commons licence, and indicate if changes were made. The images or other third party material in this article are included in the article's Creative Commons licence, unless indicated otherwise in a credit line to the material. If material is not included in the article's Creative Commons licence and your intended use is not permitted by statutory regulation or exceeds the permitted use, you will need to obtain permission directly from the copyright holder. To view a copy of this licence, visit http://creativecommons.org/licenses/by/4.0/.

\section{References}

1. Neumann F-J, Sousa-Uva M, Ahlsson A, et al. ESC/EACTS Guidelines on myocardial revascularization. EuroIntervention. 2018;2019(14):1435-534.

2. Soran O, Manchanda A, Schueler S. Percutaneous coronary intervention versus coronary artery bypass surgery in multivessel disease: a current perspective. Interact Cardiovasc Thorac Surg. 2009;8:666-71.

3. Conrotto F, Scacciatella P, D'Ascenzo F, et al. Long-term outcomes of Percutaneous coronary interventions or coronary artery bypass grafting for left main coronary artery disease in octogenarians (from a drug-eluting stent for left main artery registry substudy). Am J Cardiol. 2014;113:2007-12.

4. Posenau JT, Wojdyla DM, Shaw LK, et al. Revascularization strategies and outcomes in elderly patients with multivessel coronary disease. Ann Thorac Surg. 2017;104:107-15.

5. Hannan EL, Zhong Y, Berger PB, et al. Comparison of intermediate-term outcomes of coronary artery bypass grafting versus drug-eluting stents for patients $\geq 75$ years of age. Am J Cardiol. 2014;113:803-8.

6. Nicolini F, Contini GA, Fortuna D, et al. Coronary artery surgery versus percutaneous coronary intervention in octogenarians: long-term results. Ann Thorac Surg. 2015;99:567-74.

7. Hicks KA, Mahaffey KW, Mehran R, et al. Cardiovascular and stroke endpoint definitions for clinical trials. JAm Coll Cardiol. 2017;2018(71):1021-4.

8. Thygesen K, Alpert JS, Jaffe AS, et al. Fourth universal definition of myocardial infarction. Circulation. 2018;2018(138):e618-51.

9. Mehran R, Rao SV, Bhatt DL, et al. Standardized bleeding definitions for cardiovascular clinical trials: a consensus report from the Bleeding Academic Research Consortium. Circulation. 2011;123:2736-47.

10. Sheridan BC, Stearns SC, Rossi JS, et al. Three-year outcomes of multivessel revascularization in very elderly acute coronary syndrome patients. Ann Thorac Surg. 2010;89:1885-9.

11. Yamaji K, Shiomi H, Morimoto T, et al. Effects of age and sex on clinical outcomesafterpercutaneous coronaryintervention relative to coronary artery bypass grafting in patients with triple-vessel coronary artery disease. Circulation. 2016;133:1878-91.

12. Garcia S, Sandoval Y, Roukoz H, et al. Outcomes after complete versus incomplete revascularization of patients with multivessel coronary artery disease: a meta-analysis of 89,883 patients enrolled in randomized clinical trials and observational studies. J Am Coll Cardiol. 2013;62:1421-31.

13. Yazji K, Abdul F, Elangovan S, et al. Comparison of the effects of incomplete revascularization on 12-month mortality in patients compared with $\geq 80$ years who underwent percutaneous coronary intervention. Am J Cardiol. 2016;118:1164-70.

14. Melby SJ, Saint LL, Balsara K, et al. Complete coronary revascularization improves survival in octogenarians. Ann ThoracSurg. 2016;102:505-11.

15. Généreux P, Campos CM, Farooq V, et al. Validation of the SYNTAX revascularization index to quantify reasonable level of incomplete revascularization after percutaneous coronary intervention. Am J Cardiol. 2015;116:174-86. 